\title{
Algebraic Aspects in the Classification of $C^{*}$-Algebras
}

\author{
FRANCESC PERERA
}

\begin{abstract}
We survey some recent results concerning the use of non-stable $K$-theoretic methods to efficiently analyse the ideal structure of multiplier algebras for a wide class of $C^{*}$ algebras having real rank zero and stable rank one. Some applications of these results are delineated, showing a high degree of infiniteness of these objects.
\end{abstract}

\section{INTRODUCTION}

In the classification of $C^{*}$-algebras, $K$-Theory plays an eminent rôle which was already apparent from Elliott's pioneering work on $A F$-algebras. In a rather expository manner, we start by recalling in Section 1 the concepts of $K_{0}(A)$ (along with its order structure) and $K_{1}(A)$, for a $C^{*}$-algebra $A$. After a brief overview on the classification in general in Section 2, we turn our attention to non-stable $K$-Theory and its relation with ordered monoids. This is accomplished in Sections 3 and 4. Thus, for a wide class of non-unital simple $C^{*}$-algebras with real rank zero and stable rank one, we obtain a representation of the monoid of isomorphism classes of projections of their multiplier algebras (Theorems 4.2 and 4.3). With this representation at hand, the lattice of order-ideals in this monoid, known to be crucial for understanding the ideal structure of the multiplier algebra, can be analysed in detail. We show in Section 5 how a rich ideal structure in the multiplier algebra emerges in terms of the scale

1991 Mathematics Subject Classification. Primary 46L05, 46L80; Secondary 06F05, 46L35.

Key words and phrases. $C^{*}$-algebra, real rank, Riesz decomposition, separativity, $K$-Theory.

Partially supported by the TMR Programme of the European Commission, by the DGESIC (Spain) and by the Comissionat per Universitats i Recerca de la Generalitat de Catalunya. 
and its topological properties. The techniques introduced can also be used in Section 6 to compute the stable rank of the multiplier algebra and to examine the more general concept of extremal richness, a notion recently introduced by Brown and Pedersen in [15].

\section{Construction of $K$-Theory for $C^{*}$-algebras.}

In this section, we shall describe the construction of the $K$-groups for a given $C^{*}$-algebra $A$, with special emphasis on $K_{0}$, since this will be more relevant for our later discussion. (A much more complete discussion can be found in [9], [56] or [39].)

Let $A$ be a (unital) $C^{*}$-algebra. We say that two projections (i.e. self-adjoint idempotents) $p$ and $q$ in $A$ are Murray-von Neumann equivalent provided that there exists an element $w$ in $A$ such that $p=$ $w w^{*}$ and $w^{*} w=q$. Denote by $M_{\infty}(A)$ the directed union of $M_{n}(A)$ under the maps $M_{n}(A) \rightarrow M_{n+1}(A)$ given by $x \mapsto \operatorname{diag}(x, 0)$. We say that two projections $p$ and $q$ in $M_{\infty}(A)$ are Murray-von Neumann equivalent if they are equivalent in a suitable matrix algebra over $A$. It is easy to see that Murray-von Neumann equivalence is indeed an equivalence relation among projections in $M_{\infty}(A)$.

For a projection $p$ in $M_{\infty}(A)$, denote by $[p]$ its equivalence class, and denote by $V(A)$ the set of all equivalence classes of projections in $M_{\infty}(A)$. This is an abelian monoid under the operation $[p]+[q]=$ $[\operatorname{diag}(p, q)]$. In constructing $K_{0}$, we form a group out of $V(A)$. Since projections in $A$ need not satisfy any cancellation law, we define the cancellative monoid $V(A)_{c}$ associated to $V(A)$ by moding out by the following equivalence relation: $x \sim y$ in $V(A)$ if and only if $x+z=y+z$, for some $z$ in $V(A)$. Now the addition in $V(A)_{c}$ is defined by $\bar{x}+\bar{y}=\overline{x+y}$. This new monoid is (by construction) a cancellative one, so after adding formal inverses to the elements of $V(A)_{c}$, we define the Grothendieck group of $A$, denoted by $K_{0}(A)$, as the abelian group

$$
K_{0}(A)=\{\bar{x}-\bar{y} \mid x, y \in V(A)\} .
$$

Not only is the abelian structure of $K_{0}(A)$ relevant for the classification programme but it is also its order structure. The operation in $V(A)$ defines a natural (translation-invariant) preordering by setting $x \leq y$ if $x+z=y$, for some $z$ in $V(A)$. This preordering is referred to as the algebraic preordering. Now we equip $K_{0}(A)$ with an order structure by taking as positive cone $K_{0}(A)^{+}=\{\bar{x}-\bar{y} \mid x \leq y$ in $V(A)\}$. Note that the order in $K_{0}(A)$ is 
partial (that is, $K_{0}(A)^{+} \cap\left(-K_{0}(A)^{+}\right)=\{0\}$ ) whenever $A$ is stably finite, meaning that $x x^{*}=1$ implies $x^{*} x=1$, for any $x$ in $M_{n}(A)$ and any $n$.

A relevant concept for the order structure is that of an order-unit: if $G$ is a partially ordered abelian group with positive cone $G^{+}$, we say that a non-zero element $u$ in $G$ is an order-unit provided that for any $x$ in $G$ there exists $n$ in $\mathbb{N}$ such that $x \leq n u$. If $A$ is a $C^{*}$ algebra, the class of the unit $1_{A}$ is an order-unit for $K_{0}(A)$ (and also for $V(A))$.

To construct $K_{1}$ we use unitaries (or invertibles). A unitary in a $C^{*}$-algebra $A$ is an element $u$ in $A$ satisfying $u u^{*}=u^{*} u=1$. Let $U_{n}(A)$ denote the unitary group of $M_{n}(A)$ and let $U_{n}(A)_{0}$ be the connected component of the identity in $U_{n}(A)$. Then $K_{1}(A)$ is constructed as the inductive limit of the quotients $U_{n}(A) / U_{n}(A)_{0}$, under the maps $U_{n}(A) / U_{n}(A)_{0} \rightarrow U_{n+1}(A) / U_{n+1}(A)_{0}$ given by $u \mapsto$ $\operatorname{diag}(u, 1)$.

If $A$ is non-unital, we first construct the minimal unitization $\widetilde{A}$, which is isomorphic to $A^{+}:=A \oplus \mathbb{C}$, equipped with the pointwise sum and multiplication given by the rule

$$
(a, \lambda)(b, \mu)=(a b+\lambda b+\mu a, \lambda \mu) .
$$

The natural map $\pi: A^{+} \rightarrow \mathbb{C}$ induces a group homomorphism $\pi_{*}$ : $K_{0}\left(A^{+}\right) \rightarrow K_{0}(\mathbb{C})=\mathbb{Z}$. Then, we define $K_{0}(A)=\operatorname{Ker}\left(\pi_{*}\right)$. As to $K_{1}$, we just set $K_{1}(A)=K_{1}\left(A^{+}\right)$.

Both $K_{0}$ and $K_{1}$ are half-exact functors. Thus, given a $C^{*}$-algebra $A$ and a closed (two-sided) ideal $I$, the natural inclusion and quotient maps induce abelian group sequences:

$$
K_{i}(I) \rightarrow K_{i}(A) \rightarrow K_{i}(A / I),
$$

for $i=0,1$, which are exact in the middle. The lack of exactness at the endpoints can be measured by the so-called index and exponential maps, which turn out to link both sequences and yield the famous cyclic 6-term-exact sequence (see, e.g. [9], [56]):

$$
\begin{gathered}
K_{0}(I) \rightarrow K_{0}(A) \rightarrow K_{0}(A / I) \\
\uparrow \\
\downarrow \\
K_{1}(A / I) \leftarrow K_{1}(A) \leftarrow K_{1}(I)
\end{gathered}
$$




\section{Some APPlichtions of $K$-Theory to THE ClassificAtion OF $C^{*}$-ALGEBRAS.}

The common belief is that applications of $K$-Theory to the study of $C^{*}$-algebras started with the work of G.A. Elliott on the classification of $A F$ algebras (now already a classic result) [22]. If $A$ is a complex *-algebra, we say that $A$ is ultramatricial if $A=\cup_{n} A_{n}$, where $A_{1} \subseteq A_{2} \subseteq A_{3} \subseteq \ldots$ and each $A_{n}$ is finite-dimensional of the form

$$
A_{n}=M_{e_{n, 1}}(\mathbb{C}) \times \ldots \times M_{e_{n, k_{n}}}(\mathbb{C}),
$$

for some natural numbers $e_{i, j}$.

We say that $A$ is $A F$ ( 'approximately finite-dimensional') if $A$ contains an ultramatricial dense ${ }^{*}$-subalgebra. Equivalently, $A$ is $A F$ if $A=C^{*}-\underline{\lim } A_{n}$, where each $A_{n}$ is finite-dimensional. The classification result for $A F$ algebras is the nicest possible one in that we are not only able to give a complete isomorphism invariant, but also to compute its range. To this end, we need some more definitions.

We say that a monoid $M$ satisfies the Riesz Decomposition Property (or that $M$ is a Riesz monoid) provided that, whenever $x \leq$ $y_{1}+y_{2}$ in $M$, then there exist elements $x_{i}$ in $M$ such that $x=x_{1}+x_{2}$ and $x_{i} \leq y_{i}$ for all $i$ (see [57]). Accordingly, we say that a partially ordered abelian group $G$ is a Riesz group provided that its positive cone $G^{+}$satisfies the Riesz Decomposition Property. Finally, we say that a partially ordered abelian group $G$ is unperforated if $n x \geq 0$ implies $x \geq 0$ for $x$ in $G$ and $n$ in $\mathbb{N}$ (see, e.g. [32]).

Theorem 2.1. (Elliott, [22]) Let $A$ and $B$ be ultramatricial complex *-algebras. Then $A \cong B$ if and only if $K_{0}(A) \cong K_{0}(B)$ as ordered groups with order-units, if and only if the $A F$ algebras determined by $A$ and $B$ are isomorphic.

If $A$ is ultramatricial, then $K_{0}(A)$ is a countable Riesz, unperforated group. Conversely, if $G$ is a countable Riesz unperforated group with order-unit $u$, then there exists a unique ultramatricial algebra $A$ and an ordered group isomorphism $K_{0}(A) \cong G$ sending $\left[1_{A}\right]$ to $u$.

The next instance of interest was directed to understanding the intrinsic nature of an $A F$ algebra. Different constructions suggested that the only obstructions for being $A F$ were of $K$-theoretic nature and consisted of:

(1) having non-vanishing $K_{1}$ (while $A F$ algebras have trivial $\left.K_{1}\right)$; 
(2) having torsion on $K_{0}$.

The efforts have been therefore concentrated on studying a new invariant, $K_{*}:=K_{0} \oplus K_{1}$, and considering eventually the trace space of the algebra. This Classification Programme, known as "Elliott's Programme", addresses the class of separable, nuclear $C^{*}$-algebras with real rank zero (which properly contains the class of $A F$ algebras), with possibly some finiteness conditions such as stable finiteness.

The notion of real rank for a $C^{*}$-algebra was developed by Brown and Pedersen in 1991 (see [14]) as a non-commutative dimension theory for $C^{*}$-algebras (more suitable than the topological stable rank - see below), in the sense that the real rank of a commutative $C^{*}$-algebra $A=C(X)$ is exactly $\operatorname{dim} X$, the covering dimension of the space. The class of $C^{*}$-algebras best understood so far is the class of "zero-dimensional" ones: we say that a unital $C^{*}$-algebra $A$ has real rank zero, and we write $\operatorname{RR}(A)=0$, provided that every self-adjoint element can be approximated arbitrarily well by selfadjoint, invertible elements. In case $A$ is not unital, we say that $A$ has real rank zero provided that the minimal unitization $\widetilde{A}$ of $A$ has real rank zero. A number of equivalent formulations include the following result (see [17], [11], [14], and also [46]):

Proposition 2.2. $A C^{*}$-algebra $A$ has real rank zero if and only if for any self-adjoint element $x$ in $A$ there exists a sequence of commuting projections $e_{n}$ in $x A$ such that $x=\lim _{n} e_{n} x$.

Before attempting a large scale classification result, the first task consisted of generalizing the class of $A F$ algebras in a natural way. Naïvely, an $A F$ algebra can be written as an inductive limit of algebras of the form:

$$
M_{e_{n, 1}}(C(*)) \times \ldots \times M_{e_{n, k_{n}}}(C(*)),
$$

where $C(*)=C(*, \mathbb{C})=\mathbb{C}$. One first step is to generalize this to other spaces, thus taking into account the class of $A H$ algebras over a compact Hausdorff space $X$, which are limits (in the category of $C^{*}$-algebras) of algebras of the form

$$
M_{e_{n, 1}}(C(X)) \times \ldots \times M_{e_{n, k_{n}}}(C(X)) .
$$

As follows from work of Elliott and Evans ([26]), the irrational rotation algebras $A_{\theta}$ are $A H$ algebras over the circle which are not $A F$ (since $K_{1}\left(A_{\theta}\right)=\mathbb{Z}^{2}$ ). Recall that given an irrational $\theta$, the irrational rotation algebra $A_{\theta}$ is defined as the universal $C^{*}$-algebra 
generated by unitaries $u$ and $v$ such that $u v=e^{2 \pi i \theta} v u$. (Other examples of simple algebras arise from the work of Goodearl, see [36].) The next theorem is an amalgamation of various results that can be found in [25], [54], [29].

Theorem 2.3. If all the involved spaces are either circles (Elliott), graphs (Su), or spheres (Elliott, Gong, Lin, Pasnicu), and if the limit has real rank zero, then $K_{*}=K_{0} \oplus K_{1}$, endowed with a certain order, is a complete isomorphism invariant.

In all of the cases above, the group $K_{*}$, endowed with a certain order, satisfies a weaker form of unperforation (that allows torsion). In the simple case, this is equivalent to asking for strict unperforation on $V(A)$ (see Section 3).

These results still leave (at least) three open questions:

(1) is $K$-Theory a complete invariant for $A H$ algebras?

(2) decide whether a stably finite, separable, nuclear $C^{*}$-algebra with real rank zero is $A H$.

(3) what can we say if the limits above don't have real rank zero?

In recent years, striking progress has been made towards the solution to these questions. Also, the class of inductive limits considered has been considerably enlarged. Some of these recent results can be found in [20], [27], [12], [28] (see also [10]).

\section{Non-stable $K$-Theory. Multiplier algebras.}

Despite these good results, it might happen that $K$-Theory alone is not powerful enough for such a classification purpose. For example, Goodearl constructed in [36] a separable unital and stably finite $C^{*}$-algebra with real rank zero, and with the same $K$-Theory as an $A F$, but without being $A F$ itself. (That $C^{*}$-algebra is not $A H$ either, so that gives a negative answer to our second question above.) This strongly suggests that stable finiteness might be too mild a hypothesis, hence a somewhat stronger requirement, such as stable rank one, seems to be needed. We say that a unital $C^{*}$-algebra $A$ has (topological) stable rank one, in symbols $\operatorname{sr}(A)=1$, provided that the invertible elements of $A$ are dense in $A$. In case $A$ is not unital, then we say that $A$ has stable rank one if $\widetilde{A}$ has stable rank one (see [52]). As the notation indicates, there are higher values 
of stable rank, which have different implications in $K$-Theory. We shall address this issue later in Section 6 .

In the presence of real rank zero, stable rank one can be read in $K_{0}(A)$ by saying that the monoid $K_{0}(A)^{+}$is exactly $V(A)$. This is equivalent to having cancellation on $V(A)$ (see [11, Proposition III.2.4]). We can take this as a motivation to study the monoid $V(A)$ rather than $K_{0}(A)$ for $C^{*}$-algebras that might not have stable rank one; in fact, the construction of $K_{0}(A)$ involves a stabilization procedure that "disguises" relations among projections. Another fact supporting this point of view is that, if only real rank zero is assumed on $A$, then $V(A)$ is a Riesz monoid (by a result of Zhang, in [60]). However, this property can be lost in the passage to $K_{0}(A)$, as in fact is in some of the examples obtained by Goodearl ([36]). This is the general idea behind what has been lately called Non-stable K-Theory, developed and used in the 80's and 90 's by several authors, among them Ara, Blackadar, Brown, Goodearl, Mathieu, O'Meara, Pardo, Pedersen, Rørdam. This new setting raises the question of determining the exact nature and properties of relations of order and equivalence among projections. One important ingredient is the somewhat technical notion of strict unperforation. In detail, an abelian monoid $M$ is said to be strictly unperforated if whenever $n x+z=n y$, for some $x, y$ and $z$ in $M$ with $z \neq 0$ and $n$ in $\mathbb{N}$, then $x+w=y$, for some $w \neq 0$. If $A$ is a simple $C^{*}$ algebra, then $V(A)$ is strictly unperforated if and only if $K_{0}(A)$ is a weakly unperforated group (see [9], [24], [35]). There are no examples known among simple $C^{*}$-algebras with real rank zero and stable rank one such that $V(A)$ is not strictly unperforated, being therefore quite a natural condition to require (since it holds conceivably in all cases). We remark that, for a unital simple $C^{*}$-algebra $A$ with real rank zero, $V(A)$ is unperforated if and only if $A$ has (stable) strict comparability (see [48, Corollary 3.10]), in the sense of [8].

The class that we will subsequently focus on will be the class $\mathcal{N}$ of all simple, $\sigma$-unital, non-elementary $C^{*}$-algebras $A$ with real rank zero, stable rank one and with $V(A)$ strictly unperforated. This class strictly contains the class of $A F$ algebras, for which a considerable amount of work has been done in recent years.

We do not assume the algebras $A$ in our class to be unital. In fact, nowadays, and usually under a growing $K$-theoretic influence, the interest is centered on non-unital $C^{*}$-algebras and hence in their 
unitizations. From this point of view, the multiplier algebra of a $C^{*}$ algebra has taken an increasingly important rôle (see, for example, $[18])$.

The multiplier algebra $M(A)$ of a $C^{*}$-algebra $A$ can be characterized in several ways. It is the largest unital $C^{*}$-algebra that contains $A$ as an essential ideal. If we represent $A$ acting non-degenerately on a Hilbert space $\mathcal{H}$, then $M(A)$ is the idealizer of $A$ inside $B(\mathcal{H})$. That is,

$$
M(A)=\{x \in B(\mathcal{H}) \mid x A, A x \subseteq A\} .
$$

The corona algebra is defined as the quotient $M(A) / A$. Among the most basic examples of multiplier algebras we have that $M\left(C_{0}(X)\right)=$ $C_{b}(X)=C(\beta X)$ (where $\beta X$ is the Stone-ČCech compactification of $X)$; if $\mathcal{H}$ is a Hilbert space, then $M(K(\mathcal{H}))=B(\mathcal{H})$.

In this last example, the Calkin algebra $B(\mathcal{H}) / K(\mathcal{H})$ is simple, and that both $B(\mathcal{H})$ and $K(\mathcal{H})$ have real rank zero. For the wide class of $\sigma$-unital $C^{*}$-algebras $A$ having real rank zero and stable rank one, a sufficient condition to ensure that their multiplier algebras $M(A)$ have real rank zero is that $K_{1}(A)=0$, as proved by Lin in [44, Theorem 10]. In general, though, it is not true that if a (nonunital) $C^{*}$-algebra $A$ has real rank zero, then its multiplier algebra $M(A)$ also has real rank zero, even if $K_{1}(A)=0$ and the condition of stable rank one is dropped (see [45]). The vanishing of $K_{1}$ is merely a necessary condition for $M(A)$ to have real rank zero in case $A$ is stable (see [61]).

The study of multiplier algebras has been a common objective pursued by several authors, among them Ara, Brown, Elliott, Goodearl, Higson, Lin, Mathieu, Rørdam and Zhang (see, [21], [23], [13], [41], [58], [59], [60], [42], [43], [38], [53], [62], [63], [64], [35], [2]).

Concentrating on the multiplier algebra of a $C^{*}$-algebra $A$ in the class $\mathcal{N}$ defined before, we wish to measure its complexity by analysing the intricacy of its ideal structure. The problem we will focus on (in the next two sections) is the following:

Question: What are the ideals of $M(A) / A$, for $A$ in $\mathcal{N}$ ?

In some of the known results (especially for $A F$ algebras) concerning the lattice of ideals, no attention seems to have been directed to giving an effective description of this lattice. One of our objectives is to provide a technique which helps to accomplish such a description. This is based on formulating the problem in a more general context, of a monoid-theoretical nature, and work it out with that level of 
generality. One benefit of this approach is that we obtain simpler proofs and get some intuition on how a global picture of the ideal structure of $M(A)$ could be delineated. Further applications of our techniques can be found in (non-commutative) Ring Theory: this general framework allows to give a rather satisfactory description of multiplier rings of von Neumann regular rings (see [3], and also [4]).

\section{USING MONOIDS TO IDENTIFY IDEALS. REPRESENTATION RESULTS.}

Let $M$ be an abelian monoid. As in Section 1, we endow $M$ with the algebraic preordering. An order-ideal of $M$ is a submonoid $S$ of $M$ such that, whenever $x \leq y$, and $y \in S$, then $x \in S$ (see [5]). Given an order-ideal $S$ of a monoid $M$, we can form the quotient monoid as in [5], moding out by the following equivalence relation: if $x, y \in M$, write $x \sim y$ if and only if there exist elements $z$ and $w$ in $S$ such that $x+z=y+w$. Denote by $M / S$ the quotient modulo $\sim$, and define an addition of equivalence classes by $[x]+[y]=[x+y]$, for $x$ and $y$ in $M$. Observe that if $I$ is a closed ideal of a $C^{*}$-algebra $A$, then $V(I)$ is naturally an order-ideal of $V(A)$. We shall use $L(M)$ to denote the lattice of order-ideals of $M$, and $L_{c}(A)$ to denote the lattice of closed ideals of $A$. Results due to Zhang allow to show the following:

Theorem 4.1. ([60, Theorem 2.3]) Let $A$ be a $\sigma$-unital (non-unital) $C^{*}$-algebra with real rank zero. Then the map that assigns to each closed ideal I of $M(A)$ the order-ideal $V(I)$ of $V(M(A))$ furnishes a lattice isomorphism between $L_{c}(M(A))$ and $L(V(M(A)))$.

In view of this result, we center our efforts in studying the lattice $L(V(M(A)))$, and to do so we first relate the monoid $V(M(A))$ to the monoid $V(A)$. The underlying idea is the following: if $p$ is a projection in $M(A)$, we look at the projections in $A$ that lie "below" $p$ in a suitable sense. A precise formulation of this is given by the notion of an interval in a monoid (see [33], [37]).

An interval in an abelian monoid $M$ is a non-empty subset $I$ of $M$ that is upward directed and order-hereditary (that is, whenever $x \leq y$ and $y \in I$, then $x \in I$ ). If there exists an increasing sequence $\left(x_{n}\right)$ in $M$ such that $I=\left\{x \in M \mid x \leq x_{n}\right.$ for some $\left.n\right\}$, then we say that $I$ is countably generated (see [48]). 
Define $D(A)=\{[p] \in V(A) \mid p$ is a projection in $A\}$, for any $C^{*}$ algebras $A$. If $A$ has an approximate unit consisting of an (increasing) sequence of projections $\left(e_{n}\right)$, then $D(A)$ is an interval, countably generated by the sequence $\left(\left[e_{n}\right]\right)$.

Assume now that $A$ is $\sigma$-unital, with real rank zero and stable rank one. If $e$ is a projection in $M_{n}(M(A))$, for some $n$ in $\mathbb{N}$, denote by

$$
\theta(e)=\left\{[p] \in V(A) \mid p \text { is a projection in } e M_{n}(M(A)) e\right\} .
$$

Taking into account the fact that the hereditary subalgebra $e M_{n}(A) e$ of $M_{n}(A)$ is $\sigma$-unital ([35, Lemma 1.3]), with an increasing approximate unit $\left(e_{n}\right)$ consisting of projections ([61, Proposition 1.2]), we see that $\theta(e)$ is countably generated. In fact,

$$
\theta(e)=\left\{[p] \in V(A) \mid p \lesssim e_{k} \text { for some } k\right\} .
$$

The monoid-theoretic version of [35, Theorem 1.10] gives us the following:

Theorem 4.2. ([50, Theorem 2.4]) Let $A$ be a $\sigma$-unital $C^{*}$-algebra with real rank zero and stable rank one. Then there exists a monoid isomorphism from $V(M(A))$ onto the abelian monoid $W_{\sigma}^{D}(V(A))$ whose elements are those countably generated intervals $I$ in $V(A)$ for which there exist $n$ in $\mathbb{N}$ and a countably generated interval $J$ in $V(A)$ such that $I+J=n D$, where $D=D(A)$. This isomorphism is defined mapping every $[e]$ in $V(M(A))$ to $\theta(e)$, and it is normalized by $\theta\left(1_{M(A)}\right)=D$.

This result constitutes the first step towards the analysis of the monoid $V(M(A))$, for a $C^{*}$-algebra $A$ in the class $\mathcal{N}$ introduced in the previous section. Recall that one technical assumption for $A$ in this class consists of requiring that $V(A)$ is strictly unperforated. This provides a useful tool to analyse the ordering on the monoid via the natural representation on the state space which we describe below.

Let $K$ be a compact convex set. As usual, cf. [32], we use Aff $(K)$ to denote the group of all affine continuous real-valued functions on $K$. We denote by $\operatorname{LAff}(K)$ the monoid of all affine and lower semicontinuous functions on $K$ with values in $\mathbb{R} \cup\{+\infty\}$. Let LAff $_{\sigma}(K)$ be the submonoid of $\operatorname{LAff}(K)$ whose elements are pointwise suprema of increasing sequences of affine continuous functions on $K$. The use 
of the superscript + (respectively, ++ ) will always refer to positive (respectively, strictly positive) functions.

Let $M$ be a monoid, and let $u$ in $M$ be an order-unit. A (normalized) state on $M$ is a monoid homomorphism $s: M \rightarrow \mathbb{R}^{+}$ such that $s(u)=1$. We denote the set of (normalized) states by $S_{u}:=\operatorname{St}(M, u)$. There is a natural representation map, given by evaluation:

$$
\phi_{u}: M \rightarrow \operatorname{Aff}\left(S_{u}\right)^{+}, \phi_{u}(x)(s)=s(x) .
$$

Let $A$ be a $C^{*}$-algebra in $\mathcal{N}$, and fix a non-zero element $u$ in $V(A)$ (which will be an order-unit since $A$ is simple). Let $d=$ $\sup \phi_{u}(D(A))$, and note that $d \in \operatorname{LAff}_{\sigma}\left(S_{u}\right)^{++}$. We define the following semigroup:

$$
\begin{aligned}
& W_{\sigma}^{d}\left(S_{u}\right)=\left\{f \in \operatorname{LAff}_{\sigma}\left(S_{u}\right)^{++} \mid f+g=n d\right. \\
& \left.\quad \text { for some } g \text { in } \operatorname{LAff}_{\sigma}\left(S_{u}\right)^{++} \text {and } n \text { in } \mathbb{N}\right\} .
\end{aligned}
$$

Consider now the set $V(A) \sqcup W_{\sigma}^{d}\left(S_{u}\right)$, where we use $\sqcup$ to denote disjoint union of sets. We endow this set with a monoid structure by extending the natural operations in both $V(A)$ and $W_{\sigma}^{d}\left(S_{u}\right)$, and by setting $x+f=\phi_{u}(x)+f$, for $x$ in $V(A)$ and $f$ in $W_{\sigma}^{d}\left(S_{u}\right)$. It is not difficult to see that this is a well-defined operation, that $x+f \in W_{\sigma}^{d}\left(S_{u}\right)$ and that $d$ is an order-unit of $M \sqcup W_{\sigma}^{d}\left(S_{u}\right)$.

The next result is the key we need in order to effectively examine the ideal structure of multiplier algebras of $C^{*}$-algebras lying in the class $\mathcal{N}$. Its proof uses Theorem 4.2 as an important ingredient.

Theorem 4.3. ([50, Theorem 3.9]) Let $A$ be a $C^{*}$-algebra in $\mathcal{N}$. Fix a non-zero element $u$ in $V(A)$ and set $d=\sup \phi_{u}(D(A))$. Then there is a monoid isomorphism

$$
\varphi: V(M(A)) \rightarrow V(A) \sqcup W_{\sigma}^{d}\left(S_{u}\right),
$$

such that $\varphi([p])=[p]$ if $p \in A$ and $\varphi([p])=\sup \left\{\phi_{u}([q]) \mid[q] \in\right.$ $V(A)$ and $q \lesssim p\}$, if $p \in M(A) \backslash A$. This isomorphism is normalized by $\varphi\left(\left[1_{M(A)}\right]\right)=d$.

The function $d$ in the previous result is referred to as the scale of the algebra $A$ (cf. [41], [42]). This important fact provides a (rather systematic) procedure to analyse the lattice of ideals of multiplier algebras. It is in fact our next purpose to exploit this technique, that allows for new results as well as new insights into work previously done for $A F$ algebras. 


\section{IDEALS IN MULTIPLIER ALGEBRAS.}

In the current section, we wish to examine the interplay between the topological properties of the scale $d$ and the algebraic information of $M(A) / A$. It is natural to split our study into four cases, namely distinguishing whether the scale is continuous, finite, bounded or unbounded. By definition, and in contrast with [41], we say that $A$ has finite scale provided that $d_{\mid \partial_{e} S_{u}}$ is a finite function. Observe that this is different than requiring $d$ be globally finite, since this would immediately imply that $d$ is globally bounded (see, e.g. [50, Lemma 4.4]). This shows how a new ideal structure, previously unknown even for $A F$ algebras, opens up.

The question of determining when the corona algebra is simple has been considered in different instances (e.g. [41], [60], [42]), and was completely solved in case $A$ is simple and separable ([42, Theorem $2.10])$.

Theorem 5.1. ([42]) If $A$ is a (non-unital) simple and separable $C^{*}$-algebra, then $M(A)$ has a unique smallest closed ideal $L(A)$ that properly contains $A$. Moreover, $M(A) / A$ is simple if and only if $A$ is elementary or if the scale is continuous.

To illustrate the use of our techniques, we outline a proof of this result in case $A$ belongs to the class $\mathcal{N}$ (see also the proof of [50, Proposition 4.1]). Fix any non-zero element $u$ in $V(A)$. Let $L(A)$ be the unique closed ideal of $M(A)$ such that $V(L(A))$ is isomorphic to $L:=V(A) \sqcup \operatorname{Aff}\left(S_{u}\right)^{++}$under the isomorphism given in Theorem 4.3 (and using Theorem 4.1). Since $\operatorname{sr}(A)=1$ and $V(A) \neq 0$, we see that $K_{0}(A)$ is non-zero and partially ordered, whence $S_{u}=S t(V(A), u)$ is non-empty. Thus $L$ properly contains $V(A)$. Now, if $I$ is any order-ideal that properly contains $V(A)$, pick an element $f$ in $I$ such that $f \notin V(A)$, and take any $g$ in $\operatorname{Aff}\left(S_{u}\right)^{++}$. Since $f$ is lower semicontinuous and $g$ is continuous, there is a natural number $n$ such that $g \ll n f$. Writing $f=\sup f_{m}$, where $\left(f_{m}\right)$ is an increasing sequence in $\operatorname{Aff}\left(S_{u}\right)^{++}$, if follows by compactness (of $S_{u}$ ) that there exists $l$ in $\mathbb{N}$ such that $g \ll n f_{k}$ for all $k \geq l$. Thus $n f-g=$ $\sup _{k \geq l}\left(n f_{k}-g\right) \in \operatorname{LAff}_{\sigma}\left(S_{u}\right)^{++}$, and therefore $g \leq n f$ in $W_{\sigma}^{d}\left(S_{u}\right)$. Since $I$ is an order-ideal, we conclude that $g \in I$ whence $L \subseteq I$.

Finally, observe that $M(A) / A$ is simple if and only if $L(A)=$ $M(A)$, that is, $\operatorname{Aff}\left(S_{u}\right)^{++}=W_{\sigma}^{d}\left(S_{u}\right)$. And this is clearly equivalent to continuity of $d$. This completes the proof of the theorem. 
The next result deals with the algebras with finite scale, and at the same time answers (in the affirmative and in a wider context) the following question posed by Goodearl in 1996 ([35, Section 16]). Recall that a closed ideal $I$ in a $C^{*}$-algebra $A$ is said to be stably cofinite provided that $A / I$ is a stably finite $C^{*}$-algebra.

Question: Let $A$ be a $C^{*}$-algebra in $\mathcal{N}$ such that $\operatorname{RR}(M(A))=0$. Let $I$ be the smallest stably cofinite closed ideal of $M(A)$. Is $I=$ $L(A)$ ?

Theorem 5.2. ([50, Theorem 4.7]) Let $A$ be a separable $C^{*}$-algebra in $\mathcal{N}$. Then $A$ has finite scale if and only if $V(M(A)) / V(L(A))$ is cancellative. If, further, $\operatorname{RR}(M(A))=0$, then $A$ has finite scale if and only if $\operatorname{sr}(M(A) / L(A))=1$.

In [7, Theorem 4.8], Blackadar characterized those $A F$ algebras with bounded scale. The same criterion holds in our setting, thus extending Blackadar's result:

Theorem 5.3. (cf. [50, Theorem 5.7]) Let $A \in \mathcal{N}$. Then $A$ has bounded scale if and only if $A$ is algebraically simple.

Now we wish to study the lattice of ideals of $M(A)$ for algebras with unbounded scale. To this end, we say that a state $s$ in $S_{u}$ is infinite provided that $d(s)=\infty$.

We first consider the case when there are finitely many infinite extremal states. Observe first of all that we are restricting our attention only to the extreme boundary. Secondly, we are not requiring that the extreme boundary is finite but that there is a finite number of extreme points satisfying a given property. The case where $A$ is $A F$ and its state space is finite was considered by Elliott, Lin, Higson and Rørdam among others ([23], [41], [38]). In particular, Lin showed that in that situation the ideal structure of $M(A) / A$ can be completely described. A similar model can be adopted in our current setting, in that our methods completely describe the ideal lattice of a quotient of $M(A) / A$, giving a new insight into Lin's work, as follows.

Proposition 5.4. ([50, Proposition 6.1]) Let $A$ be a separable $C^{*}$ algebra in $\mathcal{N}$. There exists a unique closed ideal $I_{\text {fin }}(A)$ that properly contains $A$ and it is maximal among the ideals $I$ of $M(A)$ such that $V(I) / V(L(A))$ is cancellative. It can be characterized by the property

$$
V\left(I_{\text {fin }}(A)\right)=V(A) \sqcup\left\{f \in W_{\sigma}^{d} \mid f_{\mid \partial_{e} S_{u}} \text { is finite }\right\} .
$$


If, further, $M(A)$ has real rank zero, then $I_{\text {fin }}(A) / L(A)$ is the largest closed ideal of stable rank one in $M(A) / L(A)$.

Recall that a projection $p$ in a $C^{*}$-algebra $A$ is said to be infinite provided there exists a projection $q$ in $A$ such that $q<p$ and $q \sim p$. A simple $C^{*}$-algebra $A$ is said to be purely infinite if every non-zero hereditary subalgebra contains an infinite projection (see [19], and also [58]).

Theorem 5.5. (see [50, Theorem 6.3, Proposition 6.5, Theorem 6.6]) Let $A$ be a separable $C^{*}$-algebra in $\mathcal{N}$. Fix a non-zero element $u$ in $V(A)$. Let $\aleph$ be the cardinal of infinite extremal states in $S_{u}$.

(a) If $\aleph=n<\infty$, then $M(A) / I_{\text {fin }}(A)$ has exactly $2^{n}$ closed ideals, of which exactly $n$ are maximal, and exactly $n$ are minimal.

(b) If $\aleph$ is infinite, then $M(A) / I_{\text {fin }}(A)$ contains at least $\aleph$ maximal different closed ideals.

Moreover, for any such ideal $J / I_{\text {fin }}(A)$, the non-zero projections in $M(A) / J$ are infinite; and if $J$ is maximal, then $M(A) / J$ is a purely infinite simple $C^{*}$-algebra.

Despite these positive results, the intricacy of the ideal lattice of $M(A)$ is far from being completely understood. There are examples of $C^{*}$-algebras $A$ in $\mathcal{N}$ (even among $A F$ algebras) such that $M(A) / L(A)$ contains an uncountable family of different (proper) closed ideals that form a chain with respect to inclusion (in fact, these ideals can be parametrized by the open interval $(0,1))$ - see [50, Theorem 6.8]. We give another example below that exhibits a similar behaviour.

The representation technique can be refined if the extreme boundary of $S_{u}$ is a compact Hausdorff space. This occurs in several instances, and also allows for some new constructions. In this particular situation, restriction of the functions in $W_{\sigma}^{d}\left(S_{u}\right)$ to the extreme boundary of $S_{u}$ yields a monoid isomorphism:

$$
V(M(A)) \cong V(A) \sqcup W_{0}^{d}\left(S_{u}\right),
$$

where now $W_{0}^{d}\left(S_{u}\right)$ consists of all $f \in L\left(\partial_{e} S_{u}\right)^{++}$with the property that $f+g=n d_{0}$ for some $n$ and some $g$ in $L\left(\partial_{e} S_{u}\right)^{++}, L\left(\partial_{e} S_{u}\right)$ is just the semigroup of lower semicontinuous functions on $\partial_{e} S_{u}$ with values on $\mathbb{R} \cup\{+\infty\}$, and $d_{0}$ is the restriction of $d$ to the extreme boundary of $S_{u}$. What is noticeable about this fact is that the information 
is now stored in the continuity, rather that in the affinity of the functions involved.

For algebras with bounded scale, a especially rich ideal structure has been discovered in [35, Corollary 16.7]. We take this opportunity to offer an example showing that a similar pattern is also present in the unbounded scale situation. Although the existence of the family of ideals announced in the example below can be drawn from results in [49], our computations provide a direct approach and give a better description of the ideal structure.

If $X$ is a locally compact topological space and $f \in C_{b}(X, \mathbb{R})$, then $f$ can be uniquely extended to a function $f^{\beta}$ in $C(\beta X, \mathbb{R})$, by [31, Theorem 6.5]. In fact, this extension defines a ring isomorphism between $C_{b}(X, \mathbb{R})$ and $C(\beta X, \mathbb{R})$, by $[31,6.6(\mathrm{~b})]$.

Example 5.6. There exists a non-unital, simple, separable $A F$ algebra $A$ whose multiplier algebra $M(A)$ contains uncountably many maximal closed ideals between $L(A)$ and $I_{\text {fin }}(A)$.

Proof. The method of proof used is partially based on that of [35, Example 7.3, Corollary 7.4] (see also [50, Example 4.6]). Let $X=$ $[0,1]$, and let $G$ be a countable dense subgroup of $C(X, \mathbb{R})$ that contains the constant function 1 . We equip $G$ with the strict order, so that $G^{+}=\{f \in G \mid f \gg 0\} \cup\{0\}$. Let $M=G^{+}$, a cancellative simple monoid that satisfies the Riesz decomposition property and is conical (that is, the set of non-zero elements in $M$ is closed under addition). Note that 1 is an order-unit for $M$. Set $S_{1}=\operatorname{St}(M, 1)$, and note that $\partial_{e} S_{1}$ is homeomorphic to $X$. Let $d: X \rightarrow \mathbb{R}^{++} \cup\{+\infty\}$ be the lower semicontinuous function defined by $d(x)=1 / x$, for all $x$ in $X$. Since $\partial_{e} S_{1}$ is a compact space, we can extend $d$ to an affine and lower semicontinuous function on $S_{1}$, which we call $d$ again (see [35, Lemma 7.2]). Let $D=\{f \in M \mid f \ll d\}$. Then $D$ is a nonzero interval such that $\sup \phi_{1}(D)=d$. By the non-unital version of Elliott's Theorem (see [22]), there exists a (non-unital) separable, simple $A F$ algebra $A$ such that $K_{0}(A) \cong G$, and this isomorphism takes $D(A)$ to $D$. Therefore $V(A)=K_{0}(A)^{+} \cong M$, and so by the remarks preceding this example we have a monoid isomorphism

$$
\varphi: V(M(A)) \cong V(A) \sqcup W_{0}^{d}\left(S_{1}\right) .
$$

Denote $I_{\text {fin }}=\varphi\left(V\left(I_{\text {fin }}(A)\right)\right)$ and $L=\varphi(V(L(A)))$. With this notation, we have

$I_{\text {fin }}=M \sqcup\left\{f \in W_{0}^{d}\left(S_{1}\right) \mid f(0)<+\infty\right\} \quad$ and $\quad L=M \sqcup C[0,1]^{++}$. 
Let $I_{b}(A)$ be the unique closed ideal of $M(A)$ with $\varphi\left(V\left(I_{b}(A)\right)\right)=$ $M \sqcup E_{b}$, where $E_{b}=\left\{f \in W_{0}^{d}\left(S_{1}\right) \mid f\right.$ is bounded $\}$. Evidently then $L \subseteq I_{b} \subseteq I_{\text {fin }}$.

Let $f^{\prime}$ in $C[0,1]^{++}$be a continuous function such that $f \ll d$. Set $f(x)=f^{\prime}(x)$ if $x \neq 0$ and define $f(0)=\epsilon<f^{\prime}(0)$. Then the function $g:=d-f$ is lower semicontinuous and strictly positive, and $f+g=d$. Thus $f \in W_{0}^{d}\left(S_{1}\right)$, and this shows that the inclusion $L \subseteq I_{b}$ is strict.

Now, set $d^{\prime}(x)=d(x)$ if $x \neq 0$, and $d(0)=1$. Then $d^{\prime} \in L[0,1]^{++}$ and satisfies $d+d^{\prime}=2 d$. Hence the inclusion $I_{b} \subseteq I_{f i n}$ is also strict. It is clear that $I_{\text {fin }}$ is a proper order-ideal of $M \sqcup W_{0}^{d}\left(S_{1}\right)$.

We now describe the quotient $I_{b} / L$. Denote by $\iota:\{0\} \sqcup E_{b} \rightarrow I_{b}$ the natural inclusion. Observe that $\iota\left(\{0\} \sqcup C[0,1]^{++}\right) \subset L$, and hence we can define a monoid homomorphism:

$$
\bar{\iota}:\left(\{0\} \sqcup E_{b}\right) /\left(\{0\} \sqcup C[0,1]^{++}\right) \rightarrow I_{b} / L,
$$

in the natural way. It is easy to see that $\bar{\iota}$ is indeed an isomorphism.

Denote by $B=C_{b}((0,1], \mathbb{R})$ the ordered vector space of all bounded, real valued, continuous functions defined on $(0,1]$ and let $C=$ $\left\{f \in B \mid \lim _{t \rightarrow 0} f(t)=0\right\}$, which is a convex subgroup of $B$. Define

$$
\varsigma:\left(\{0\} \sqcup E_{b}\right) /\left(\{0\} \sqcup C[0,1]^{++}\right) \rightarrow B / C
$$

by setting $\varsigma(0)=0$ and $\varsigma([f])=\left[f_{\mid(0,1]}-f(0)\right]$. Note that $\varsigma$ is a monoid homomorphism, and that $\varsigma(x)=0$ if and only if $x=0$. We now show that $\varsigma$ is injective. Assume that $f, g \in E_{b}$ for which there exist functions $h_{1}, h_{2}$ in $C$ such that $f_{\mid(0,1]}-f(0)+h_{1}=$ $g_{\mid(0,1]}-g(0)+h_{2}$. Since $(f-f(0)-g+g(0))_{\mid(0,1]}=h_{2}-h_{1}$ and $\lim _{t \rightarrow 0}\left(h_{2}-h_{1}\right)(t)=0$, we see that in fact $f-g$ is continuous on $[0,1]$.

Thus, there exist continuous functions $l_{1}$ and $l_{2}$ in $C[0,1]^{++}$such that $f-g=l_{2}-l_{1}$. Therefore $[f]=[g]$ in $\left(\{0\} \sqcup E_{b}\right) /\left(\{0\} \sqcup C[0,1]^{++}\right)$, so $\varsigma$ is injective, as claimed.

Let $f \in E_{b}$. We claim that $f_{\mid(0,1]}-f(0) \in B^{+}+C$. Define an upper semicontinuous function $g$ on $[0,1]$ by $g(0)=f(0)$ and $g(x)=0$ for $x \neq 0$. Then $g \leq f$ and hence by the Sandwich Theorem (e.g. [30, 1.7.15]) there exists a function $h$ in $C([0,1], \mathbb{R})$ such that $g \leq h \leq f$. Note that $f(0)=h(0)$. Let $k:=h_{\mid(0,1]}-h(0)$. Then $k \in C$ and $\left(f_{\mid(0,1]}-f(0)\right)-k=f_{\mid(0,1]}-h_{\mid(0,1]} \geq 0$, whence $\left(f_{\mid(0,1]}-f(0)\right)-k \in$ $B^{+}$, establishing the claim. Therefore

$$
\varsigma\left(\left(\{0\} \sqcup E_{b}\right) /\left(\{0\} \sqcup C[0,1]^{++}\right)\right) \subseteq\left(B^{+}+C\right) / C .
$$


To see that in fact we have equality, take $h$ in $B^{+}$. Then there exists $f$ in $L[0,1]^{++}$such that $f_{\mid(0,1]}=h+1$ and $f(0)=1$. Since $h \leq m$ for some $m$ in $\mathbb{N}$ and $d \geq 1$, there exists a natural number $n$ such that $f \ll n d$. Define $g(x)=(n d-f)(x)$ if $n \neq 0$ and $g(0)=\infty$. Then $g \in L[0,1]^{++}$and $f+g=n d$, whence $f \in W_{0}^{d}\left(S_{1}\right)$. Note that in fact $f \in E_{b}$ and $\varsigma([f])=\left[f_{\mid(0,1]}-1\right]=[h]$.

We have proved that the monoids $I_{b} / L$ and $\left(B^{+}+C\right) / C=(B / C)^{+}$ are isomorphic. Recall that $B$ is isomorphic to $C(\beta(0,1], \mathbb{R})$ by extending each function $f$ in $B$ to $f^{\beta}$. If $x \in \beta(0,1]$, define $I_{x}=\{f \in$ $\left.B \mid f^{\beta}(x)=0\right\}$. Then $\left(I_{x}\right)_{x \in \beta(0,1]}$ is a family of different maximal ideals of $B$, by [31, Theorem 7.2]. Moreover $C \subseteq I_{x}$ for any element $x$ in $\beta(0,1] \backslash(0,1]$. Finally, note that $\beta(0,1] \backslash(0,1]$ is uncountable. Indeed, since $(0,1] \cong \mathbb{R}^{+} \cong \mathbb{R}^{-}$, we have that

$$
\beta(0,1] \backslash(0,1] \cong \beta \mathbb{R}^{+} \backslash \mathbb{R}^{+} \cong \beta \mathbb{R}^{-} \backslash R^{-},
$$

and

$$
\beta \mathbb{R} \backslash \mathbb{R} \cong\left(\beta \mathbb{R}^{+} \backslash \mathbb{R}^{+}\right) \cup\left(\beta \mathbb{R}^{-} \backslash R^{-}\right),
$$

according to $[31,6.10]$. Finally, $[55,4.45]$ ensures that $\beta \mathbb{R} \backslash \mathbb{R}$ is uncountable. Therefore the group $B / C$ contains uncountably many different maximal ideals, which have the form $I_{x} / C$, where $x \in \beta(0,1] \backslash(0,1]$. Consequently, $\left(I_{x} / C\right) \cap(B / C)^{+}$are different maximal ideals of the monoid $(B / C)^{+}$, which correspond to ideals of $I_{b} / L$ (under $\bar{\iota} \circ \varsigma^{-1}$ ), and this finishes the proof.

\section{Applications: stable Rank and extremal Richness.}

In this final section, we will show further applications of the techniques and results in the previous sections.

The first application refers to the computation of stable rank. If $A$ is a (unital) $C^{*}$-algebra, denote by $L g_{n}(A)=\left\{\left(a_{1}, \ldots, a_{n}\right) \mid\right.$ $\left.\sum_{i=1}^{n} A a_{i}=A\right\}$, where $n \geq 1$. The (topological) stable rank of $A$ is the smallest integer $n$ such that $L g_{n}(A)$ is dense in $A^{n}$ (or $\infty$ if no such $n$ exists). We write $\operatorname{sr}(A)=n$. If $A$ is non-unital, then by definition $\operatorname{sr}(A)=\operatorname{sr}(\widetilde{A})$ (see [52]). This number is of importance in $K$-Theory since it gives an estimation of the smallest $n$ such that $K_{1}(A)$ can be "generated" by $U_{n}(A)$ (cf. [6]).

A well-known result of Rieffel ([52, Proposition 6.5]) ensures that whenever a unital $C^{*}$-algebra $A$ has two isometries with orthogonal ranges, then $\operatorname{sr}(A)=\infty$. This happens, for instance, when 
$A=B(\mathcal{H})$, where $\mathcal{H}$ is an infinite dimensional separable Hilbert space. Zhang showed ([58, Corollary 1.6]) that this is also the case for $M(A) / A$, where $A$ is $\sigma$-unital with real rank zero and continuous scale. These phenomena would seem to preclude any stable rank finiteness conditions either on $M(A)$ or on $M(A) / A$. We show that this is not the case in general, and that the scale of $A$ is again the main tool that provides enough information to compute the stable rank. In particular, our techniques give a partial negative answer to the following:

Question: (Rieffel, 1983) Are there finiteness conditions to be imposed on $M(A)$ (or on $A$ itself) such that $\operatorname{sr}(M(A))=\operatorname{sr}(A)$ ?

The key to the result that we present below is separativity. This is a weak cancellation condition that can be defined in a purely monoidtheoretical setting: an abelian monoid $M$ is said to be separative if whenever $a+a=a+b=b+b$, for $a$ and $b$ in $M$, then $a=b$ (see [16]). A $C^{*}$-algebra $A$ is called separative if the corresponding monoid $V(A)$ is a separative monoid (see [5]). (It is also said that $A$ has stable weak cancellation.) The importance of this condition relies on the fact that it provides a key to a number of cancellation problems in (non-commutative) Ring Theory and also in Operator Algebras (see [5]). In fact, there are no examples known of $C^{*}$-algebras with real rank zero whose $V(A)$ 's are not separative. Finding such an example is one major challenge in the area.

In [5] Ara, Goodearl, O'Meara and Pardo proved that if $A$ is a unital separative $C^{*}$-algebra $A$ with real rank zero, then the only possible values for $\operatorname{sr}(A)$ are 1,2 or $\infty$. We make use of this result in the proof of the following:

Theorem 6.1. ([50, Theorem 7.5]) Let $A$ be a separable $C^{*}$-algebra in $\mathcal{N}$. Then $V(M(A))$ is separative. If, further, $M(A)$ has real rank zero, then $\operatorname{sr}(M(A))=2$ if and only $A$ has finite but not continuous scale, and $\operatorname{sr}(M(A))=\infty$ otherwise.

Corollary 6.2. ([50, Corollary 7.6]) Let $A$ be a separable $C^{*}$-algebra in $\mathcal{N}$. Assume that $\operatorname{RR}(M(A))=0$. Then $\operatorname{sr}(M(A))=2$ if and only if $\operatorname{sr}(M(A) / A)=2$.

So far, multiplier algebras have appeared to us as rather infinite objects from various points of view. In particular, we see from the last results that their stable rank will never be equal to one. The rest of the section deals with the concept of extremal richness, which may be regarded as a generalized stable rank one. This notion was 
introduced by Brown and Pedersen in [15] with the objective of extending the theory and results for stable rank one $C^{*}$-algebras to the infinite case. A unital $C^{*}$-algebra $A$ is extremally rich provided that the quasi-invertible elements are dense in $A$. Here, an element $x$ in $A$ is quasi-invertible if, by definition, $x \in A^{-1} E(A) A^{-1}$, where $E(A)$ is the set of extreme points of the closed unit ball of $A$. By [47, Proposition 1.4.7], we have $E(A)=\left\{x \in A \mid\left(1-x^{*} x\right) A\left(1-x x^{*}\right)=0\right\}$. Extremal richness is well-behaved under the passage to quotients, hereditary subalgebras (in particular, ideals), formation of matrices and other natural constructions (see [15]). We shall consider the following problem:

Question: Are $M(A)$ or $M(A) / A$ extremally rich?

This question was previously considered by Larsen and Osaka in [40], for a variety of classes of $C^{*}$-algebras. For example, they proved that, if $A$ is a purely infinite simple $C^{*}$-algebra ( $\sigma$-unital and nonunital), then $M(A)$ is extremally rich. In case $A$ is simple, separable and $A F$, and if it has a finite number of extremal (semifinite) traces, of which at least two are infinite, then $M(A) / A$ is not extremally rich. If, on the other hand, $A$ has only one infinite extremal trace, then $M(A) / A$ is extremally rich.

Our methods allow to solve this problem within our class $\mathcal{N}$, thus enlarging the number of instances where extremal richness of the multiplier and corona algebras can be analysed. The approach to this problem combines the analysis of extremal richness under extensions (see [15] and [40]) with the description of the ideal structure of $M(A)$ given in the previous sections.

We shall use the notation $L Q T_{d}(A)$ to denote the convex set of densely defined, lower semicontinuous quasitraces on $A$. Denote by $K(A)$ the minimal dense ideal of $A$ (see [47, Theorem 5.6.1]). If $p$ is a non-zero projection of $A$, then denote by

$$
Q_{p}=\left\{\tau \in L Q T_{d}(A) \mid \tau_{\mid K(A)_{+}}<\infty \text { and } \tau(p)=1\right\},
$$

which is a weak* compact set if $A$ is simple. If moreover $A$ is $\sigma$-unital and has real rank zero, then the natural map

$$
\alpha: Q_{p} \rightarrow S t(V(A),[p]), \alpha(\tau)([q])=\tau(q),
$$

is an affine homeomorphism. This is the semifinite version of Blackadar-Handelman's Theorem in [11, Theorem III.1.3] (see [50, Theorem $5.6]$ for a proof of how this case is obtained from the unital case.). We say in this context that a quasitrace $\tau$ in $Q_{p}$ is infinite provided that 
$\sup \tau(q)=\infty$, where $q$ runs over the set of projections of $A$ (equivalently, its corresponding state under the map $\alpha$ described above is infinite, in the terminology of the previous section). In particular, all the results in Section 5 can be rephrased making reference only to quasitraces on $A$, instead of states on $V(A)$. Observe in the next results that again we are not requiring any finiteness assumption on the cardinality of the extremal quasitraces.

Theorem 6.3. ([51, Theorem 2.12]) Let $A$ be a separable $C^{*}$-algebra in $\mathcal{N}$, and let $p$ be a non-zero projection in $A$.

(a) If $A$ has finite scale and $\operatorname{RR}(M(A))=0$, then $M(A) / A$ is extremally rich .

(b) If $A$ has at least two infinite extremal quasitraces in $Q_{p}$, then $M(A) / A$ is not extremally rich. In particular, $M(A)$ is not extremally rich .

Theorem 6.4. ([51, Theorem 3.12, Corollary 3.13]) Let $A$ be a separable $C^{*}$-algebra in $\mathcal{N}$ such that $\operatorname{RR}(M(A))=0$, and let $p$ be a non-zero projection in $A$. Suppose that $A$ has exactly one infinite extremal quasitrace $\tau$ in $Q_{p}$. Then $M(A) / A$ is extremally rich if and only if the complementary face of $\{\tau\}$ in $Q_{p}$ is closed. If, further, $\partial_{e} Q_{p}$ is compact, then $M(A) / A$ is extremally rich if and only if $\{\tau\}$ is an isolated point in $\partial_{e} Q_{p}$.

By adapting an argument of [40] to our setting, we conclude that $M(A)$ is never extremally rich, for a $C^{*}$-algebra $A$ in the class $\mathcal{N}$ (see [51, Proposition 3.14]).

\section{ACKNowledGement}

This article comprises two talks delivered at the meeting $C^{*}$-Algebras at Queen's in Belfast on May 28th, 1999. The author congratulates Martin Mathieu on the organization of a fine meeting.

\section{REFERENCES}

1. C.A. Akemann, G.K Pedersen, J. Tomiyama, Multipliers of $C^{*}$-algebras, J. Funct. Anal. 13 (1973), pp. 277-301.

2. P. Ara, M. Mathieu, A simple local multiplier algebra, Math. Proc. Cambridge Phil. Soc., 126 (1999), pp. 555-564.

3. P. Ara, F. Perera, Multipliers of von Neumann regular rings, Comm. in Algebra, 28 (7), (2000), pp. 3359-3385.

4. P. Ara, E. Pardo, F. Perera, The structure of countably generated projective modules over regular rings, J. Algebra, 226 (2000), pp. 161-190. 
5. P. Ara, K.R. Goodearl, K.C. O'Meara, E. Pardo, Separative cancellation for projective modules over exchange rings, Israel J. Math., 105 (1998), pp. 105-137.

6. H. Bass, "Introduction to some methods of algebraic $K$-Theory", Regional Conference Series in Mathematics, 21, A.M.S., Providence, R.I., 1974.

7. B. Blackadar, Traces on simple $A F C^{*}$-algebras, J. Funct. Anal., 38 (1980), pp. $156-168$.

8. B. Blackadar, Comparison theory for simple $C^{*}$-algebras, in "Operator Algebras and Applications" (D. Evans and M. Takesaki, eds.), London Math. Soc. Lecture Notes, Vol. 135, 1988, pp. 21-54.

9. B. Blackadar, " $K$-Theory for operator algebras", Springer Verlag, New York, 1986.

10. B. Blackadar, Classification of nuclear $C^{*}$-algebras by $K$-Theory, Summer school in Operator Algebras (Lecture Notes), Odense, 1996.

11. B. Blackadar, D. Handelman, Dimension functions and traces on $C^{*}$ algebras, J. Funct. Anal., 45 (1982), pp. 297-340.

12. O. Bratteli, G.A. Elliott, D.E. Evans, A. Kishimoto, On the classification of $C^{*}$-algebras of real rank zero. III. The infinite case. Operator algebras and their applications, II (Waterloo, ON, 1994/1995), pp. 11-72, Fields Inst. Commun., 20, Amer. Math. Soc., Providence, R.I., 1998.

13. L.G. Brown, Semicontinuity and multipliers of $C^{*}$-algebras, Canad. J. Math., 40 (1988), pp. 865-988.

14. L.G. Brown, G.K. Pedersen, $C^{*}$-algebras of real rank zero, J. Funct. Anal., 99 (1991), pp. 131-149.

15. L.G. Brown, G.K. Pedersen, On the geometry of the unit ball of a $C^{*}$-algebra, J. reine angew. Math., 469 (1995), pp. 113-147.

16. A.H. Clifford, G.B. Preston, "The algebraic theory of semigroups", Vol. 1, Math. Surveys, 7, A.M.S., Providence, R. I., 1961.

17. J. Cuntz, The structure of multiplication and addition in simple $C^{*}$-algebras, Math. Scand., 40 (1977), pp. 215-233.

18. J. Cuntz, A class of $C^{*}$-algebras and topological Markov chains, II, Invent. Math., 63 (1981), pp. 25-40.

19. J. Cuntz, $K$-Theory for certain $C^{*}$-algebras, Ann. of Math., 113 (1981), pp. 181-197.

20. S. Eilers, A complete invariant for $A D$ algebras with real rank zero and bounded torsion in $K_{1}$, J. Funct. Anal., 139 (1996), pp. 325-348.

21. G.A. Elliott, Derivations of matroid $C^{*}$-algebras II, Ann. of Math., 100 (1974), pp. 407-422.

22. G.A. Elliott, On the classification of inductive limits of sequences of semisimple finite-dimensional algebras, J. Algebra, 38 (1976), pp. 29-44.

23. G.A. Elliott, The ideal structure of the multiplier algebra of an $A F$ algebra, C. R. Math. Rep. Acad. Sci. Canada, 9 (1987), pp. 225-230.

24. G.A. Elliott, Dimension groups with torsion, Int. J. Math., 1 (1990), pp. 361-380.

25. G.A. Elliott, On the classification of $C^{*}$-algebras of real rank zero, J. reine angew. Math., 443 (1993), pp. 179-219.

26. G.A. Elliott, D.E. Evans, The structure of the irrational rotation $C^{*}$-algebra, Ann. of Math., 138 (1993), pp. 477-501. 
27. G.A. Elliott,G. Gong, On the classification of $C^{*}$-algebras of real rank zero. II, Ann. of Math. (2) 144 (1996), pp. 497-610.

28. G.A. Elliott, G. Gong, H. Su, On the classification of $C^{*}$-algebras of real rank zero. IV. Reduction to local spectrum of dimension two. Operator algebras and their applications, II (Waterloo, ON, 1994/1995), pp. 73-95, Fields Inst. Commun., 20, Amer. Math. Soc., Providence, R.I., 1998.

29. G.A. Elliott, G. Gong, H. Lin, C. Pasnicu, Abelian $C^{*}$-subalgebras of $C^{*}$-algebras of real rank zero and inductive limit $C^{*}$-algebras, Duke Math. $J ., 85$ (1996), pp. 511-554.

30. R. Engelking, "General Topology", Rev. and Compl. Ed., Heldermann Verlag, Berlin, 1989.

31. L. Gillman, M. Jerison, "Rings of continuous functions", Van Nostrand, Princeton, 1960; Graduate Texts in Mathematics, No. 43. Springer-Verlag, New York-Heidelberg, 1976 (Reprint).

32. K.R. Goodearl, "Partially ordered abelian groups with interpolation", Math. Surveys and Monographs, 20, A.M.S., Providence, R. I., 1986.

33. K.R. Goodearl, Extensions of dimension groups and $A F C^{*}$-algebras, $J$. reine angew. Math., 412 (1990), pp. 150-219.

34. K.R. Goodearl, Notes on a class of simple $C^{*}$-algebras with real rank zero, Publ. Matem., 36 (1992), pp. 637-654.

35. K.R. Goodearl, $K_{0}$ of multiplier algebras of $C^{*}$-algebras with real rank zero, $K-$ Theory, 10 (1996), pp. 419-489.

36. K.R. Goodearl, $C^{*}$-algebras of real rank zero whose $K_{0}$ 's are not Riesz groups, Canad. Math. Bull., 39 (4) (1996), pp. 429-437.

37. K.R. Goodearl, D.E. Handelman, Stenosis in dimension groups and $A F C^{*}$ algebras, J. reine angew. Math., 332 (1982), pp. 1-98.

38. N. Higson, M. Rørdam, The Weyl-von Neumann theorem for multipliers of some AF algebras, Canad. J. Math. 43 (1991), pp. 322-330.

39. F. Larsen, N. Laustsen, M. Rørdam, "An introduction to $K$-Theory for $C^{*}$-algebras", London Mathematical Society Student Texts, 49, 2000.

40. N. Larsen, H. Osaka, Extremal richness of multiplier algebras of corona algebras of simple $C^{*}$-algebras, J. Operator Theory, 38 (1997), pp. 131-149.

41. H. Lin, Ideals of multiplier algebras of simple $A F C^{*}$-algebras, Proc. Amer. Math. Soc., 104 (1988), pp. 239-244.

42. H. Lin, Simple $C^{*}$-algebras with continuous scales and simple corona algebras, Proc. Amer. Math. Soc., 112 (3) (1991), pp. 871-880.

43. H. Lin, Notes on $K$-Theory of multiplier algebras and corona algebras. Preprint.

44. H. Lin, Exponential rank of $C^{*}$-algebras with real rank zero and the BrownPedersen conjectures, J. Funct. Anal., 114 (1993), pp. 1-11.

45. H. Lin, H. Osaka, Real rank of multiplier algebras of $C^{*}-$ algebras of real rank zero, Operator algebras and their applications (Waterloo, ON, 1994/1995), pp. 235-241, Fields Inst. Commun., 13, Amer. Math. Soc., Providence, R.I., 1997.

46. P. Menal, Spectral Banach algebras with bounded index, J. Algebra, 154 (1) (1993), pp. 27-66.

47. G.K. Pedersen, " $C^{*}$-algebras and their automorphism groups", Academic Press, London/New York, 1979. 
48. F. Perera, The structure of positive elements for $C^{*}$-algebras with real rank zero, Int. J. Math., 8 (3), (1997), pp. 383-405.

49. F. Perera, "Teoria- $K$ no estable per a anells de multiplicadors", Doctoral Thesis, Universitat Autònoma de Barcelona, 1998.

50. F. Perera, Ideal structure of multiplier algebras of simple $C^{*}$-algebras with real rank zero, Canad. J. Math., to appear.

51. F. Perera, Extremal richness of multiplier and corona algebras of simple $C^{*}$ algebras with real rank zero, J. Operator Theory, 44 (2000), pp. 413-431.

52. M.A. Rieffel, Dimension and stable rank in the $K$-Theory of $C^{*}$-algebras, Proc. London Math. Soc., 46 (3) (1983), pp. 301-333.

53. M. Rørdam, Ideals in the multiplier algebra of a stable $C^{*}$-algebra, J. $O p$ erator Theory, 25 (1991), pp. 283-298.

54. H. Su, $K$-theoretic classification for certain real rank zero $C^{*}$-algebras, Quantum and non-commutative analysis (Kyoto, 1992), pp. 387-397, Math. Phys. Stud., 16, Kluwer Acad. Publ., Dordrecht, 1993.

55. R.C. Walker, "The Stone-Čech compactification", Erg. der Math. und ihrer Grenzg., Vol 83, Springer-Verlag, Berlin, 1974.

56. N.E. Wegge-Olsen, " $K$-Theory and $C^{*}$-algebras", Oxford Univ. Press, 1993.

57. F. Wehrung, Monoids of intervals of ordered abelian groups, J. Algebra, 182 (1996), pp. 287-328.

58. S. Zhang, On the structure of projections and ideals of corona algebras, Canad. J. Math., Vol. 41 (1989), pp. 721-742.

59. S. Zhang, $C^{*}$-algebras with real rank zero and the internal structure of their corona and multiplier algebras, Part III, Canad. J. Math., 42 (1990), pp. $159-190$.

60. S. Zhang, A Riesz decomposition property and ideal structure of multiplier algebras, J. Operator Theory, 24 (1990), pp. 209-225.

61. S. Zhang, $K_{1}$-groups, quasidiagonality, and interpolation by multiplier projections, Trans. Amer. Math. Soc., 325 (1991), pp. 793-818.

62. S. Zhang, $C^{*}$-algebras with real rank zero and their corona and multiplier algebras, Part I, Pacific J. Math., 155 (1992), pp. 169-197.

63. S. Zhang, $C^{*}$-algebras with real rank zero and their corona and multiplier algebras, Part II, K-Theory, 6 (1992), pp. 1-27.

64. S. Zhang, $C^{*}$-algebras with real rank zero and their corona and multiplier algebras, Part IV, Int. J. Math., 3 (1992), pp. 309-336.

Francesc Perera,

Departament de Matemàtiques,

Universitat Autònoma de Barcelona,

E-08193 Bellaterra (Barcelona), Spain

perera@mat.uab.es

Received on 1 December 1999. 